\title{
Intersystem Soft Handover for Converged DVB-H and UMTS Networks
}

\author{
Xiaodong Yang and Thomas J. Owens
}

\begin{abstract}
Digital video broadcasting for handhelds (DVB-H) is the standard for broadcasting Internet Protocol (IP) data services to mobile portable devices. To provide interactive services for DVB-H, the Universal Mobile Telecommunications System (UMTS) can be used as a terrestrial interaction channel for the unidirectional DVB-H network. The converged DVB-H and UMTS network can be used to address the congestion problems due to the limited multimedia channel accesses of the UMTS network. In the converged network, intersystem soft handover between DVB-H and UMTS is needed for an optimum radio resource allocation, which reduces network operation cost while providing the required quality of service. This paper deals with the intersystem soft handover between DVB-H and UMTS in such a converged network. The converged network structure is presented. A novel soft handover scheme is proposed and evaluated. After considering the network operation cost, the performance tradeoff between the network quality of service and the network operation cost for the intersystem soft handover in the converged network is modeled using a stochastic tree and analyzed using a numerical simulation. The results show that the proposed algorithm is feasible and has the potential to be used for implementation in the real environment.
\end{abstract}

Index Terms-Compressed mode, digital video broadcasting for handhelds (DVB-H), handover, stochastic trees, Universal Mobile Telecommunications System (UMTS).

\section{INTRODUCTION}

D IGITAL video broadcasting for handhelds (DVB-H) [1], which is a digital broadcast standard, is targeting mainly Internet Protocol (IP) data services, called IP Datacast. It is assumed that a large number of the IP Datacast services will be interactive in nature [2]. A DVB-H network is a unidirectional broadcasting network that provides a means to deliver large quantities of popular contents to large user groups at relatively low cost but is very limited in providing interactivity and personalization of contents. A Universal Mobile Telecommunications System (UMTS) network, as a cellular telecommunications network, does not provide a low-cost delivery mechanism for simultaneously downloading large quantities of data to large

Manuscript received September 7, 2005; revised July 26, 2006, April 22, 2007, and September 10, 2007. This work was supported in part by the Information Society Technologies Sixth Framework Programme Integrated Project INSTINCT under Contract 507014, funded by the European Commission. The review of this paper was coordinated by Prof. Y.-B. Lin.

X. Yang was with the University of Göttingen, 37073 Göttingen, Germany, and was also with Hutchison 3G Austria GmbH, 1110 Vienna, Austria (e-mail: eepgxxy@yahoo.co.uk).

T. J. Owens is with Brunel University, UB8 3PH Uxbridge, U.K. (e-mail: Thomas.owens@brunel.ac.uk).

Color versions of one or more of the figures in this paper are available online at http://ieeexplore.ieee.org.

Digital Object Identifier 10.1109/TVT.2007.909278 user groups but has the excellent properties of interactivity and personalization. UMTS is, therefore, an excellent system to support IP Datacast by providing a terrestrial interaction channel for DVB-H. In such a converged network, DVB-H works as a high-bit-rate download channel, whereas UMTS provides the interaction channel for DVB-H. Another application area of such a converged network is the so-called "hotspot" area, where the UMTS network cannot provide the requested data delivery services because of its limited data channels. In this case, DVB-H can provide an alternative way to deliver the requested data delivery services to relieve the congested UMTS cells.

DVB-H will broadcast the same data services to as many users as possible within its coverage area. When only a few users request the same service, using DVB-H as a downlink channel will waste bandwidth that could be utilized by many more users. In this case, UMTS is an efficient way to provide the service to the limited number of users. On the other hand, when the number of users requiring the same service exceeds a certain limit, DVB-H will be a profitable way to provide the downlink channel. The intersystem handover in this paper is not defined as service continuation when a user moves out of one network-covered area but as the optimum radio resource reallocation between UMTS and DVB-H when the user is in an area that is covered by both UMTS and DVB-H networks. The scenario of the users moving out of the range of one technology will not be discussed in this paper. The service of the converged network that is referred to in this paper is the IP data delivery, as it is a service that can exploit the intersystem handover in an easily described way. Because the handover in DVB-H is always soft handover and because of the better performance of the soft handover compared with the hard handover in service continuation [3], [4], this paper focuses on the intersystem soft handover in the converged network.

So far, intersystem handover studies for UMTS have only focused on the handover between UMTS and the Global System for Mobile Communications (GSM) [5], [6] and between UMTS and wireless local area networks (WLANs) [7], [8]. No literature is available concerning the handover between UMTS and DVB-H in a converged UMTS/DVB-H network at the time of the writing of this paper. In this paper, an intersystem soft handover scheme is presented for the converged network and evaluated using a Markov chain model. Since network operation cost is also an issue in the converged network, a stochastic tree model is used to analyze the handover scheme when considering the network operation cost and the network QoS.

The rest of this paper is organized as follows. Section II provides the background of the research work. Section III 
provides an overview of the proposed converged network structure and of the intersystem handover issue. Section IV presents the intersystem soft handover algorithm between UMTS and DVB-H, including handover measurement, handover criteria, and handover execution and evaluation. In Section $\mathrm{V}$, the stochastic tree concept is introduced into the communication area, a stochastic tree model is set up to analyze the handover scheme, and a numerical simulation is presented. The optimum tradeoff between the converged network operation cost and the QoS is obtained from the simulation results. Section VI concludes this paper.

\section{RESEARCH BACKGROUND}

Although there are no previous concrete works regarding the convergence of DVB-H and UMTS, some relevant discussions have already been made in various works in the literature.

Reference [9] presents a signaling protocol for the exchange of information between a network management system and intelligent multimodal wireless terminals in a composite radio network, where different radio networks are available, and some preliminary measurement works were done mainly between WLANs, General Packet Radio Service (GPRS), and digital video broadcasting for terrestrials (DVB-T). Although the idea of redistributing terminals over different radio networks was pointed out in [9], the description was very simple, and it did not present how such redistribution would be performed in detailed steps.

Reference [10] presents two typical heterogeneous wireless network scenarios with DVB-T, WLANs, and GPRS. The first scenario refers to the redistribution of traffic between DVB-T and other available access technologies. The second scenario refers to the users moving out of the multicoverage regions and having only DVB-T access available. However, it lacks the detailed procedures on how the redistribution in the first scenario is triggered and performed.

Reference [11] focuses on the integration of WLANs and GPRS by describing how the two networks are interworking each other, mainly from the architectural point of view, while omitting the radio resource optimization part. Reference [12] proposed a way for the UMTS network operators to forecast the traffic of the high-demand services and transfer them to a cooperating DVB-T network when appropriate. However, no detailed procedures of transferring the DVB-T traffic to UMTS are described.

While [5]-[8] focus on how the intersystem handover is actually performed between either UMTS and GSM or UMTS and WLANs, [9]-[12] focus on the radio resource optimization between the broadcast and the telecommunications networks. Furthermore, all the aforementioned works did not consider the tradeoff between the network operation cost and the network QoS, and no quantization of such parameters is available.

Based on the thoughts of the previous works, this paper tries to combine both of the above two ways of thinking by proposing a novel intersystem soft handover algorithm between DVB-H and UMTS. Although this paper describes the manner in which the intersystem handover should be performed on the terminal part, just like those briefly mentioned in [13], more space in this paper is allotted for the radio resource optimization part of the intersystem handover algorithm. Whereas the compressed mode in UMTS and the time slicing mode in DVB-H are utilized for the handover process, the load factor is proposed as the threshold-based approach criterion, and a novel stochastic tree algorithm is proposed for the optimum radio resource allocation part in a proposed clear and practical converged network architecture. As an obvious evolution of the previous work, the network operation cost and the network QoS are quantized and simulated. For simplicity and focus reasons, the service continuation scenario in the converged networks that is caused by the users moving out of one network coverage area will not be considered in this paper.

\section{Converged Network Overview}

Multitier networks that provide coverage to the same areas using several cells of different sizes are useful to accommodate high traffic density while providing high QoS [14]. The proposed converged DVB-H and UMTS network cell structure is a hierarchical cell structure in which the DVB-H cells overlay the UMTS cells. Therefore, the converged network can be regarded as a multitier network from the cell-structure point of view, as shown in Fig. 1.

In the converged DVB-H and UMTS network, the DVB-H cells cover the same area as the UMTS cells do. Because the DVB-H cell size is usually larger than the UMTS cell size, one DVB-H cell normally covers several UMTS cells. Hence, the converged network has a two-layer macro-micro cell structure, in which the DVB-H cell is in the macro layer, whereas the UMTS cell is in the micro layer. This overlapping cell structure of the converged network is very useful in a "hotspot" area, which is defined as an area where the traffic load is substantially larger than the designed load [15], such as a sports arena, a concert stadium, and a theatre.

In order for the terminal to be able to perform handover between DVB-H and UMTS, either the terminal has two independent receivers, or the terminal has a single dual-mode receiver. For handheld devices, it will reduce complexity and cost to have a single dual-mode receiver rather than have two independent receivers. The user equipment (UE) referred to in this paper is assumed to be such a dual-mode handheld terminal.

In Fig. 1, the downlink stream from the Playout Server has two paths to reach the UE. One is through the DVB-H network; the other one is through the downlink channels in UMTS. The uplink stream packets from the UE have only one path, which is the UMTS uplink channel. In the converged network, the DVB-H network and the UMTS network have a common core network, which is the IP network. It is important to note that the data in the DVB-H network are IP packets and not MPEG2 packets like those in the DVB-T network. There is also a Service Management Server (SMS), which is located in the IP core network. The SMS is the key component in charge of the radio resource allocation and the intersystem soft handover between DVB-H and UMTS.

Handover within the converged network takes three forms: 1) handover within the DVB-H network; 2) handover within 


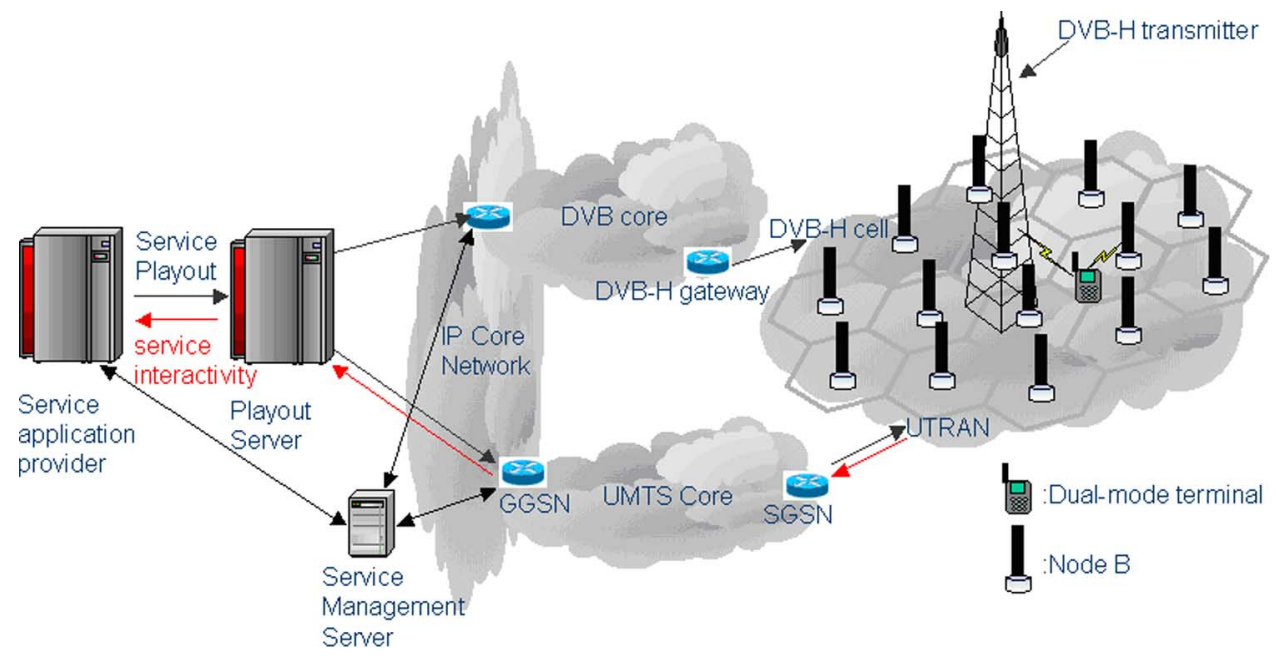

Fig. 1. Converged DVB-H and UMTS network structure.

the UMTS network; and 3) the intersystem handover between DVB-H and UMTS. This paper only considers the intersystem handover between DVB-H and UMTS.

The intersystem handover between UMTS and DVB-H in the converged network is different from the intersystem handover between UMTS and other radio access networks like GSM. In the DVB-H/UMTS converged network, there is always an uplink channel from the UE to the IP core network through the UMTS network. Therefore, the handover between UMTS and DVB-H only refers to the downlink channel handover that should have no significant effects on the uplink channels of the UE. In this intersystem soft handover scheme, the downlink channel in UMTS only refers to the dedicated channel (DCH) because only the DCH can support soft handover in UMTS. Another difference from other intersystem handover schemes is that the intersystem handover in a DVB-H/UMTS converged network is triggered not only by technical issues like physical constraints and a better radio resource allocation but also by economic issues like the network operation cost. The network cost issue is important for the intersystem handover in a converged DVB-H/UMTS network because the converged network is most likely jointly operated by a broadcasting network operator and a telecommunications network operator.

\section{HANdover Between UMTS AND DVB-H}

The intersystem soft handover mechanism between UMTS and DVB-H in the converged network can be divided into the following three stages: 1) handover measurement; 2) handover decision based on the handover criteria; and 3) handover execution.

\section{A. Performing DVB-H Measurements With the Compressed Mode of UMTS}

UMTS has two modes-the frequency division duplex (FDD) mode and the time division duplex mode. For simplicity, only the FDD mode is considered in this paper. In the FDD mode, the UE is continuously receiving on the UMTS downlink channel. This normal continuous mode is not suitable for intersystem measurement that needs transmission gaps. The
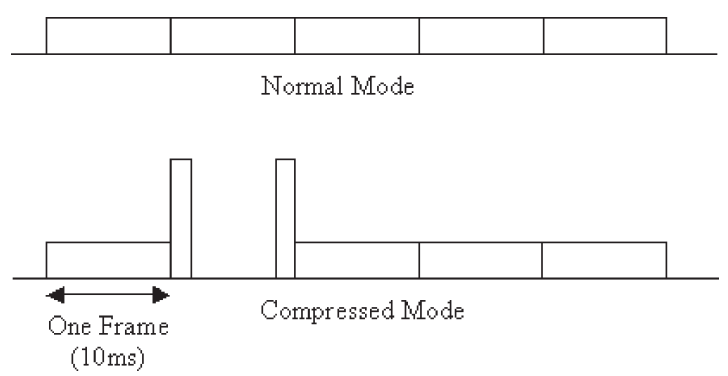

Fig. 2. Comparison between the normal mode and the compressed mode.

compressed mode, which was introduced in the 3rd Generation Partnership Project standard [16], has, therefore, been proposed to perform the intersystem handover between UMTS and GSM [5], [6].

UMTS transmits data using 10-ms-long frames. Each frame consists of 15 time slots. In the compressed mode, some frames are compressed. In the compressed frames, some slots are not used for transmitting data, thus creating transmission gaps. The instantaneous transmit power is increased in the compressed frame to keep the quality unaffected by the reduced processing gain. The compressed mode and the normal mode frame structures are compared in Fig. 2. In the compressed mode, the first few milliseconds and the last few milliseconds in a frame are used to transmit all the information in the frame, and the remaining duration of the frame is used to carry out the intersystem soft handover measurement. In this way, when performing the soft handover from UMTS to DVB-H, the compressed mode is used to create transmission gaps as measurement periods for the terminal to make the required measurements of DVB-H network parameters.

\section{B. Performing UMTS Measurements With the Time Slicing Mode of DVB-H}

The time slicing mode was introduced in the DVB-H standard [1] to reduce the average power consumption of the terminal and enable smooth seamless soft handover. In the time slicing mode, the data packets are sent in periodic bursts using significantly higher instantaneous bit rates compared to 

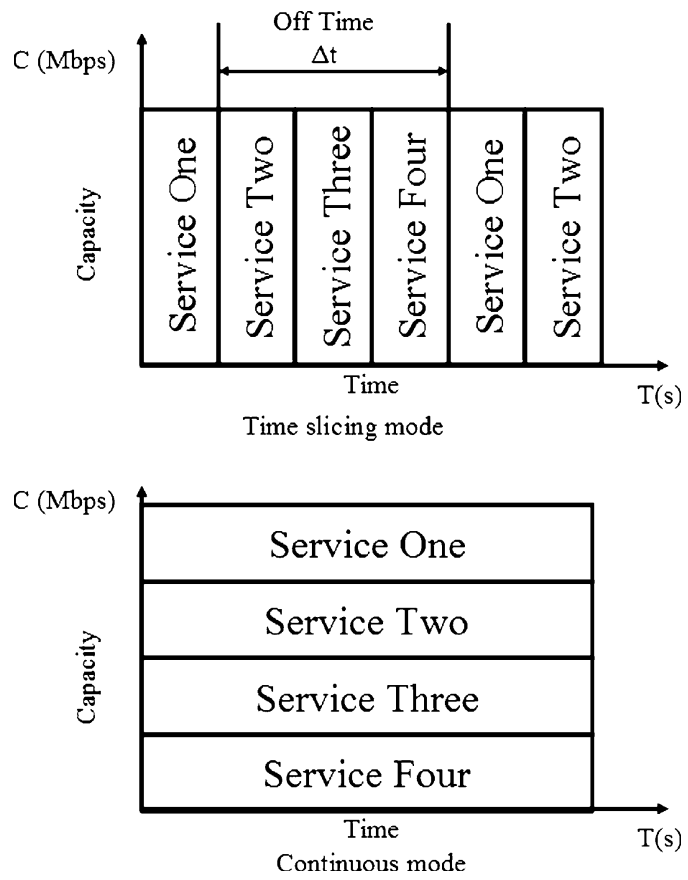

Fig. 3. Comparison between the continuous mode and the time slicing mode.

the bit rates that are required if the data were transmitted using the continuous mode like that of DVB-T [17]. The continuous mode and the time slicing mode are illustrated in Fig. 3. When performing the handover from DVB-H to UMTS, the UE carries out the handover measurement of UMTS network parameters using the off burst time of the time slicing mode. Because the intersystem handover measurement always happens in the transmission gaps for UMTS and DVB-H, the handover between the two networks is soft handover and can be seamless.

\section{Intersystem Soft Handover Criteria}

The intersystem handover between UMTS and DVB-H in the converged network is both a technical issue and an economic issue, and this must be accounted for in choosing the handover criteria. The intersystem soft handover criteria are considered only from the network side here. For the implementation of the soft handover in the terminal, the signal strength or the signal-to-noise ratio has also to be used as a handover criterion [3], [4].

Two different approaches can be taken to measure the load of the UMTS air interface. The first defines the load via the received and transmitted wideband power; the second is based on the sum of the bit rates that are allocated to all currently active subscribers [18]. The throughput-based loading status is chosen for the handover from the UMTS network part to be coherent with the handover criteria of the DVB-H network part. The UMTS downlink load factor is estimated based on the sum of the bit rates of all the currently active connections in a cell divided by the specified maximum throughput for the same cell, i.e.,

$$
\eta_{D L}=\left(\sum_{k=1}^{N} R_{k}\right) / R_{\max }
$$

where $R_{k}$ is the bit rate of connection $k$, and $N$ is the total number of connections in DCHs in one cell. $R_{\max }$ is the maximum allowed throughput of the DCH in the cell. Since the UMTS soft handover can only be supported in the DCH [18], the downlink bit rates from the forward access channels and the downlink shared channels will not be considered. The UMTS load factor must be kept below the threshold value, i.e.,

$$
\eta_{\text {oldDL }}+\Delta \eta \leq \eta_{\text {thresholdDL }}
$$

where $\eta_{\text {oldDL }}$ is the UMTS network load before the user request, which is estimated using (1), $\eta_{\text {thresholdDL }}$ is the preset load threshold, and $\Delta \eta$ is the load increase after a new connection is set up.

If the air interface loading is allowed to excessively increase, the QoS of the existing connections cannot be guaranteed. This will trigger the handover process. However, even when the UMTS network is not heavily loaded, it can still be profitable to the handover to DVB-H. The threshold for the handover, thus, should be based on economic and technical analyses. The broadcast company and the telecommunications company have to jointly make a decision on the criteria for the handover. Details of the tradeoff between the economic and technical issues are discussed later in this paper. In this case, besides the satisfaction of the load status from (2), the handover algorithm for UMTS has to decide whether the number of users receiving the same service increases above a threshold

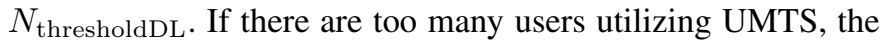
UMTS network will inform the SMS to switch these users to DVB-H. The algorithm concerning threshold $N_{\text {thresholdDL }}$ can be expressed as

$$
N_{\text {oldDL }}+\Delta L_{\text {umts }} \leq N_{\text {thresholdDL }}
$$

where $N_{\text {oldDL }}$ is the number of subscribers requesting the same data service in UMTS before a new subscriber joins. $N_{\text {thresholdDL }}$ is the preset subscriber number threshold, and $\Delta L_{\text {umts }}$ is the increase in the number of subscribers for the specific data service. $N_{\text {thresholdDL }}$ will be obtained later in this paper using the stochastic tree model.

On the other hand, the downlink load factor in DVB-H is expressed by

$$
\theta_{\mathrm{DL}}=\left(\sum_{i=1}^{M} P_{i}\right) / P_{\max }
$$

where $P_{i}$ is the bit rate of data channel $i, M$ is the total number of data channels in the DVB-H cell, and $P_{\max }$ is the maximum allowed data throughput in the DVB-H cell. Before considering the DVB-H side handover criteria, the DVB-H load factor must be kept below the threshold value, i.e.,

$$
\theta_{\text {oldDL }}+\Delta \theta \leq \theta_{\text {thresholdDL }}
$$

where $\theta_{\text {oldDL }}$ is the DVB-H network load before the user request, $\theta_{\text {thresholdDL }}$ is the preset load threshold, and $\Delta \theta$ is the load increase after a new data channel is used to accommodate new users. 

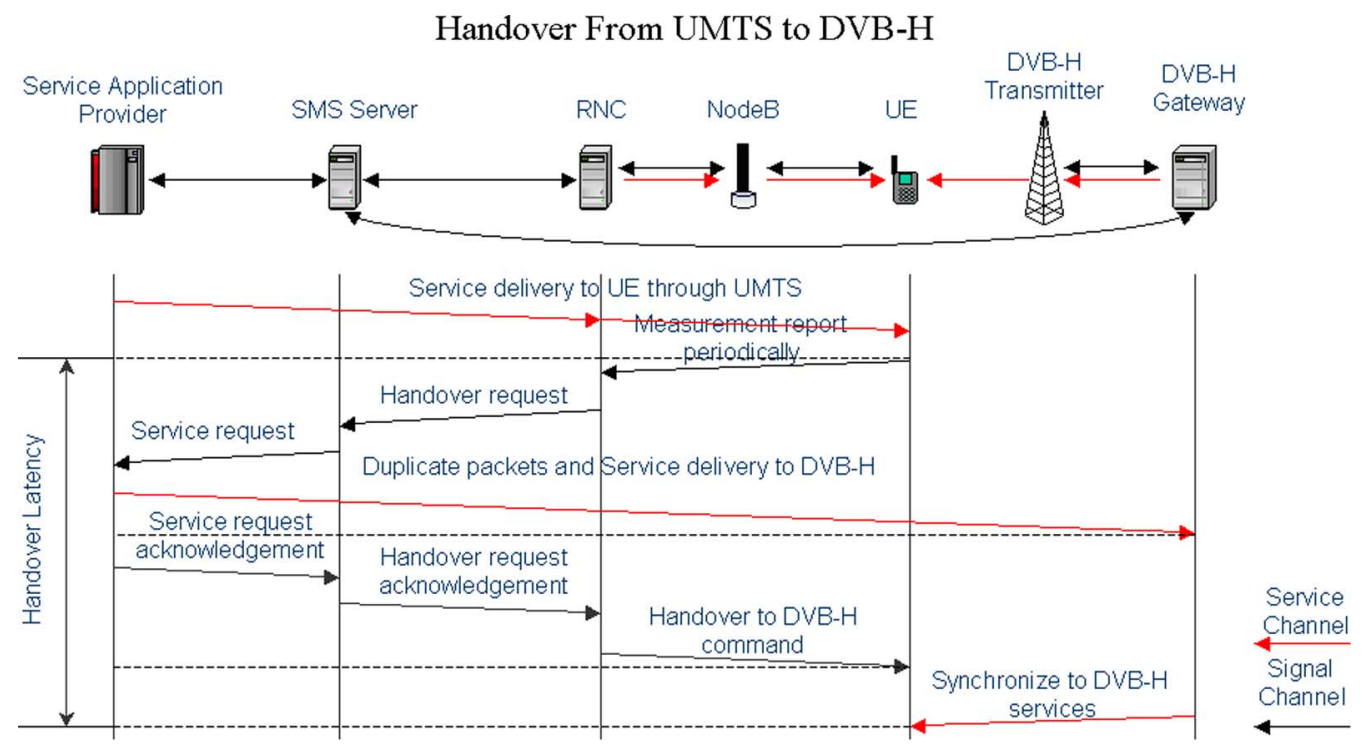

Fig. 4. Handover procedure from UMTS and DVB-H.

From the DVB-H side, when a subscriber leaves or enters the DVB-H network, besides the satisfaction of the load status from (5), the handover algorithm for DVB-H has to decide whether the number of users receiving the same service drops below a threshold $M_{\text {thresholdDL. If there are too few users utilizing }}$ DVB-H, the DVB-H network will inform the SMS to switch these users to UMTS. The algorithm concerning threshold $M_{\text {thresholdDL }}$ can be expressed as

$$
M_{\mathrm{oldDL}}-\Delta L_{\mathrm{dvbh}} \geq M_{\text {thresholdDL }}
$$

where $M_{\mathrm{oldDL}}$ is the number of subscribers requesting the same data service in DVB-H before a subscriber leaves the DVB-H network. $M_{\text {thresholdDL }}$ is the preset subscriber number threshold for one channel, and $\Delta L_{\mathrm{dvbh}}$ is the decrease in the number of subscribers for the specific data service. The number of subscribers in the DVB-H network can be obtained from the UMTS network. Furthermore, $M_{\text {thresholdDL }}$ will be obtained later in this paper using the stochastic tree model.

Equations (2), (3), (5), and (6) denote the intersystem soft handover criteria.

\section{Handover Execution Between UMTS and DVB-H}

The intersystem soft handover is a network-initiated and mobile-assisted handover scheme. The handover algorithm execution between DVB-H and UMTS is performed through the cooperation of the two networks. In the converged network, the SMS is the key component for the intersystem soft handover management between UMTS and DVB-H. It makes the final decision for the intersystem soft handover. The handover procedures are described as follows.

The first scenario is the handover from UMTS to DVB-H, as shown in Fig. 4. Suppose that the UE is receiving a service delivery from UMTS. The Radio Network Controller (RNC) within the UMTS Terrestrial Radio Access Network (UTRAN) periodically receives a measurement report from the
UE through Node B (UMTS Base Station). When the RNC finds any existing UE requesting a new service or a new UE requesting to join in the UMTS network, it will make a handover judgment based on the handover criteria and the measurement report from the UE. If the decision is to hand over the UE to DVB-H, the RNC will send handover request messages to the SMS. If the SMS accepts the handover request from UMTS, it will send service request messages to the service application provider (SAP). Then, the SAP sends service request acknowledgement messages back to the SMS. The SMS will then send the handover request acknowledgement messages back to the RNC. Before the SAP sends service request acknowledgement messages to the SMS, it will duplicate and deliver the requested service to the targeted DVB-H gateway through DVB-H. Furthermore, the DVB-H gateway will deliver the requested services to the UE. When the RNC receives the handover request acknowledgement messages from the SMS, it sends handover commands to the UE. The UE then releases resources from UMTS and performs handover to DVB-H.

The second scenario is the handover from DVB-H to UMTS, as shown in Fig. 5. In this scenario, it is supposed that the UE is receiving a file delivery from the DVB-H network. When the number of UEs in the DVB-H cell falls below the preset threshold value, this information in the periodic reports is transmitted through Node B to the RNC. The RNC will make a handover judgment based on the handover criteria and the measurement reports from each of the UEs. If the decision is to hand over the UEs from DVB-H to UMTS, the RNC will send handover request messages to the SMS. If the SMS accepts the handover requests from UMTS, it will send service request messages to the SAP. Then, the SAP will send service request acknowledgement messages back to the SMS. The SMS will then send the handover request acknowledgement messages back to the RNC. Before the SAP sends service request acknowledgement messages to the SMS, it will duplicate and deliver the requested service to the targeted RNC through the UMTS network. Furthermore, the RNC will unicast 

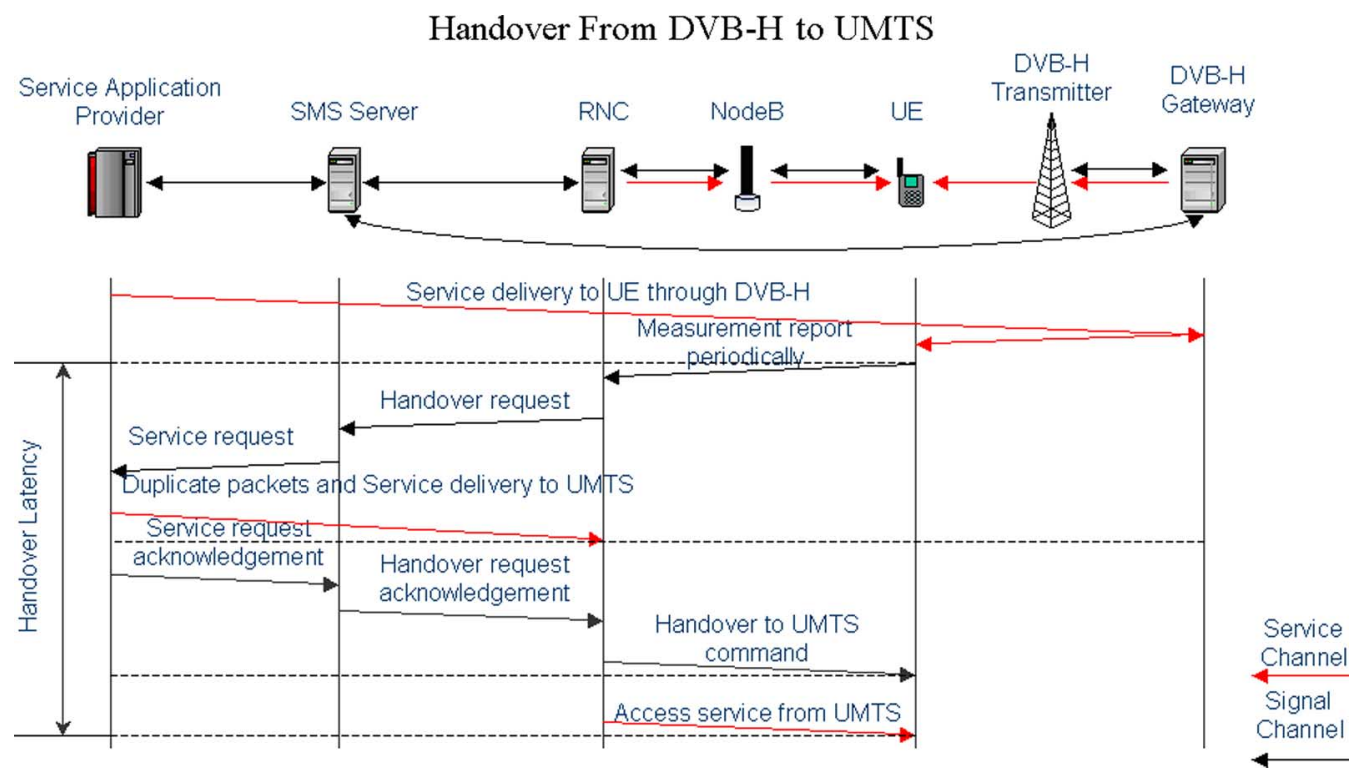

Fig. 5. Handover procedure from DVB-H to UMTS.

the requested services to the targeted UE. When the RNC receives the handover request acknowledgement messages from the SMS, it sends handover commands to the UE. The UE then releases resources from DVB-H and performs the handover to UMTS.

\section{E. Handover Performance Evaluation}

There are a lot of performance parameters that need to be evaluated for this intersystem soft handover algorithm, such as the handover latency, the packet loss, and the handover failure probability. The handover latency is the time duration from when the UE sends the periodic measurement report, which contains the handover triggering information to the RNC, until the UE gets access to the targeted service either from the UMTS or DVB-H network, as shown in Figs. 4 and 5. From Figs. 4 and 5, it can be seen that the targeted service is delivered toward the UE before the UE hands over to the targeted network. Although such service duplication adds overhead to the network, the handover can be considered seamless, with a negligible packet loss. In this section, only the handover failure probability of the proposed handover algorithm is evaluated, as space does not allow all the parameters to be evaluated in this paper, and handover failure is the most fundamental limitation on the proposed algorithm. The other parameters will be evaluated in a subsequent work.

Handover failure happens when the user needs to hand over to the targeted network according to the handover criteria but fails to do so mainly because the targeted network resources are all occupied and cannot provide more channels to accommodate more users. A Markov chain model is used to evaluate the handover failure probability of the proposed handover algorithm here.

A UMTS and DVB-H overlap cell area is taken as an example analysis area. To simplify the analysis, it is assumed that there are a maximum of $m$ data channels in DVB-H and a maximum of $n$ data channels in UMTS in the area. One channel

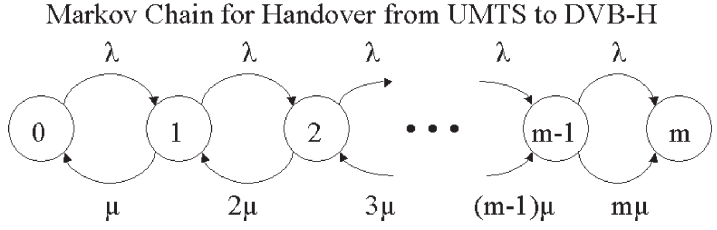

Fig. 6. Markov chain for handover from UMTS to DVB-H.

refers to one stream of a service delivery. The handover from UMTS to DVB-H and from DVB-H to UMTS are separately analyzed.

When handover from UMTS to DVB-H happens, one or several channels of UMTS will be released, and only one DVB-H channel will be occupied. The DVB-H data delivery channels can be modeled by an $\mathrm{M} / \mathrm{M} / \mathrm{m} / \mathrm{m}$ queuing process. The state transition diagram is shown in Fig. 6, where state $j$ means that there are $j$ busy DVB-H channels in the area. $\lambda_{j}$ and $\mu_{j}$ are the birth rate and the death rate at state $j$, respectively, i.e.,

$$
\begin{aligned}
\lambda_{j} & =\lambda, \quad j=0,1,2, \ldots, m-1 \\
\mu_{j} & =j_{\mu}, \quad j=1,2, \ldots, m .
\end{aligned}
$$

When $0 \leq j<m$, a state $j$ will change to $j+1$ if a handover to the DVB-H request arrives in the area. Similarly, when a handover to the UMTS request has arrived in the area, the state $j(j>0)$ will change to $j-1$. According to Erlang's loss formula [19], the handover from UMTS to DVB-H failure probability in the area is

$$
P_{\text {failure }}=P(m)=\frac{A^{m}}{m !} / \sum_{j=0}^{m} \frac{A^{j}}{j !}
$$

where $P(m)$ is the steady-state probability that the system is at state $m$, and $A=\lambda / \mu$ is the offered traffic intensity.

When a group of users in UMTS request an already broadcast service in DVB-H, they will be handed over to 


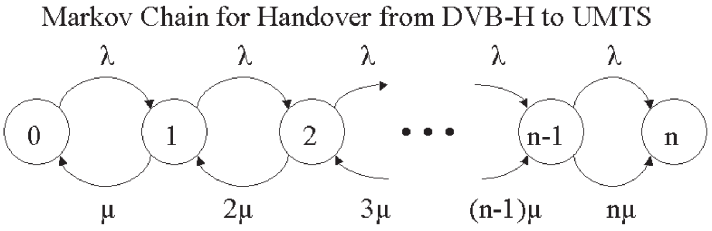

Fig. 7. Markov chain for handover from DVB-H to UMTS

DVB-H without occupying any new DVB-H channels. Such users do not have a handover failure problem; therefore, they are not considered in the above model.

When the handover from DVB-H to UMTS happens, one or several channels of UMTS will be occupied, and only one DVB-H channel will be released. The UMTS data delivery channels can be modeled by an $\mathrm{M} / \mathrm{M} / \mathrm{n} / \mathrm{n}$ queuing process. The state transition diagram is shown in Fig. 7, where state $i$ means that there are $i$ busy UMTS channels in the area. $\lambda_{i}$ and $\mu_{i}$ are the birth rate and the death rate at state $i$, respectively, i.e.,

$$
\begin{aligned}
& \lambda_{i}=\lambda, \quad i=0,1,2, \ldots, n-1 \\
& \mu_{i}=i_{\mu}, \quad j=1,2, \ldots, n .
\end{aligned}
$$

Similarly to the handover from UMTS to DVB-H, according to Erlang's loss formula [19], the handover from DVB-H to UMTS failure probability in the area is given by

$$
P_{\text {failure }}^{\prime}=P(n)=\frac{A_{1}^{n}}{n !} / \sum_{j=0}^{n} \frac{A_{1}^{i}}{i !}
$$

where $P(n)$ is the steady-state probability that the system is at state $n$, and $A_{1}=\lambda / \mu$ is the offered traffic intensity. Therefore, the total intersystem soft handover failure probability $P$ is given by

$$
P=a P_{\text {failure }}+b P_{\text {failure }}^{\prime}=a \frac{A^{m}}{m !} / \sum_{j=0}^{m} \frac{A^{j}}{j !}+b \frac{A_{1}^{n}}{n !} / \sum_{i=0}^{n} \frac{A_{1}^{n}}{i !}
$$

where $a$ is the intersystem soft handover probability of users from UMTS to DVB-H, and $b$ is the intersystem soft handover probability of users from DVB-H to UMTS.

Reasonable values are now assigned to the parameters in (11) to obtain some numerical results to indicate the handover failure probability of the proposed algorithm. It is assumed that $n=80$ (several UMTS cells) and that $m=100$ (one DVB-H cell in the area). The traffic intensities in the area are assumed to be high at $A=50$ and $A_{1}=50$. The intersystem soft handover failure probability, which is also called the handover blocking probability, is usually acceptable when it does not exceed $2 \%$ [20]. A diagram illustrating (11) is given in Fig. 8.

From Fig. 8, it can easily be seen that the maximum intersystem soft handover failure probability is much less than $2 \%$. With the same methods, even when the traffic intensity grows to $A=A_{1}=69$, as shown in Fig. 9, the maximum handover failure probability is still acceptable at $2 \%$. It can also be seen that in the $n=80, m=100$ scenario, the maximum acceptable

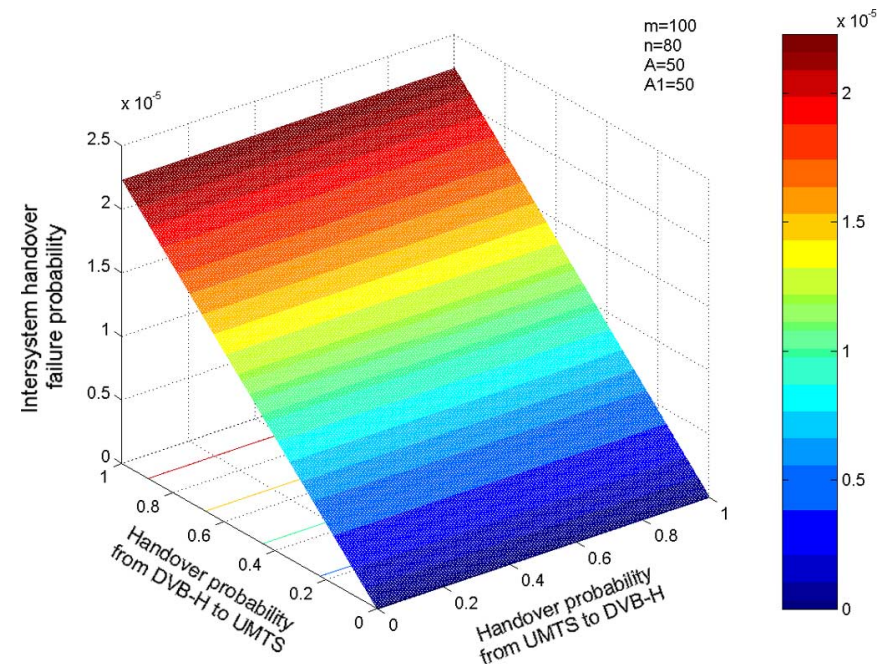

Fig. 8. Intersystem soft handover failure probability $\left(A=A_{1}=50\right)$.

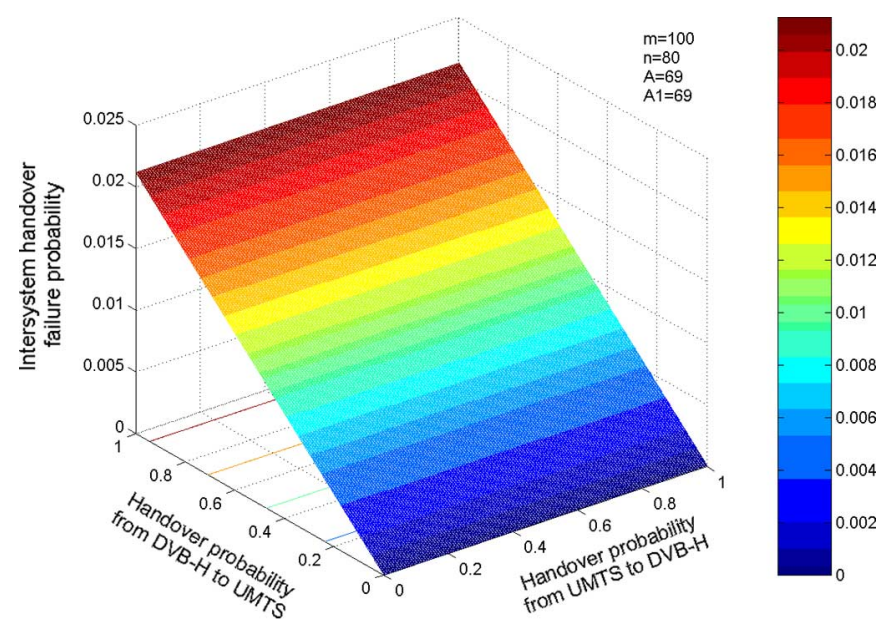

Fig. 9. Intersystem soft handover failure probability $\left(A=A_{1}=69\right)$.

traffic intensity is $A=69$ and $A_{1}=69$, if $A=A_{1}$. Using (11), the intersystem soft handover failure probability in any scenario can be determine, provided that the necessary parameters are known.

\section{Stochastic Tree Model And Analysis}

The above sections presented the intersystem soft handover algorithm. Since maintaining the converged network connection and the QoS levels and reducing the network operation cost when the subscriber requests begin are all crucial, the costs of network operation must be integrated into the handover algorithm by the network administration. To find out whether a user should use DVB-H or UMTS in the scenario of a given service delivery, the tradeoff between the user received QoS and the network operation cost must be identified. When the optimum tradeoff is found, the threshold value of the handover criteria can be optimized. With this objective in mind, the stochastic tree concept is introduced to analyze the performance of the intersystem soft handover between UMTS and DVB-H in a converged network. 


\section{A. Stochastic Tree Instead of the Multidimensional Markov Chain With Loops}

The multidimensional Markov chain with loops has long been used in various works in the literature to mainly calculate the call blocking probability of the state with multiple channels, where the effect of mobility is very well expressed [15], [20], [21]. However, the multidimensional Markov chain with loops cannot be used to model the cost parameter of the state, where the cost is a temporal factor that is increasing in the time domain and is not reversible. Instead, stochastic trees are extensions of decision trees that facilitate the modeling of temporal uncertainties [22], [23]. It is an efficient modeling approach for the decision problems in which the cost and the QoS may extend over time. On one hand, this paper focuses on the radio resource optimization part of the converged networks, concentrating on the cost and the QoS progress in the time domain. On the other hand, in the proposed network structure in Section III, the users will always have available access to UMTS and DVB-H networks. Thus, user mobility is not a concern in the considered scenario. This way, to consider the radio resource optimization decisions on the network side instead of focusing on the user mobility on the terminal side, stochastic trees are used instead of the multidimensional Markov chains with loops.

\section{B. Stochastic Tree Model for the Converged Network}

In its simplest and most useful form, a stochastic tree is a transition diagram for a continuous-time Markov chain, which is unfolded into a tree structure. Researchers have already used stochastic trees as modeling tools to analyze medical treatment decisions for almost a decade [24]. In this paper, for the first time, stochastic trees are being used in the communications field to make handover decisions to predict the converged network operation cost and the network QoS that can be expected, given an initial network state and an established handover plan. An advantage of the stochastic tree model is that it allows the recursive computation of the mean quality-adjusted duration, which is the mean duration with quality weights, by "rolling back" the stochastic tree much as one would roll back a decision tree [25]. Before applying the stochastic tree model to our intersystem handover process, the Markovian rollback formula will be developed for this case. Detailed information about the use of the Markovian rollback formula in a medical decision process is provided in [25]. The development of the Markovian rollback formula for the converged network is shown below.

Consider a subtree of a stochastic tree shown in Fig. 10 in which an initial state $x$ is occupied until one of several competing transitions with rates $\lambda_{y}$ occurs at a state $y$. The nodes represent states, initialization, UMTS, DVB-H, soft handover, and so on, and the arrows represent transitions between states. There are two types of arrows that are used in stochastic trees corresponding to the two types of possible transitions. Wavy arrows are labeled with rates and signify transitions that take time to accomplish. Straight arrows are labeled with probabilities and signify an immediate transition to one of the states indicated. There are only wavy arrows in Fig. 10. Using

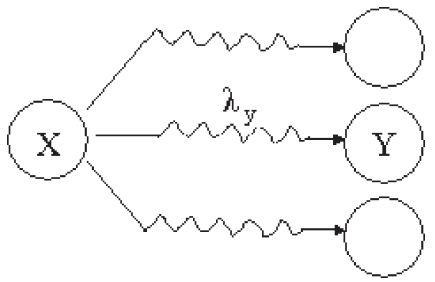

Fig. 10. Stochastic subtree.

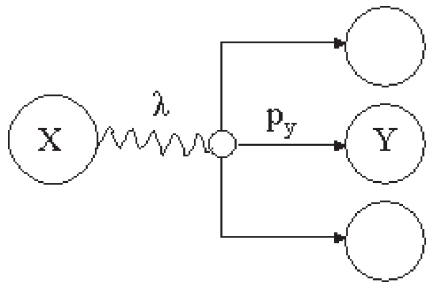

Fig. 11. Stochastic subtree transformation.

the wavy and straight arrows representation, the subtree in Fig. 10 can be transformed into the subtree in Fig. 11, where $p_{y}=\lambda_{y} / \lambda$, and $\lambda=\sum_{y} \lambda_{y}$. It is apparent that beginning with state $x$, a mean time $1 / \lambda$ is spent in state $x$, following which, the transition to a state $y$ occurs with probability $p_{y}$.

To model the intersystem soft handover in the converged network, a new concept called the quality service time (QAST) is introduced. This network measurement is defined as being calculated by weighting each time interval $T_{x}$ that is spent in a particular network state $x$ by a received service quality factor $v(x)$ that is proportional to the service quality estimation of that network state, i.e.,

$$
\mathrm{QAST}=\sum_{x} v(x) T_{x}
$$

where a service quality factor $v(x)=1$ refers to perfect reception, whereas $v(x)=0$ refers to no reception; $v(x)$ takes values between 0 and 1 . Suppose that the QAST beginning in state $y$ is $S(y)$. Then, the QAST beginning at $x$ is given by

$$
\begin{aligned}
S(x) & =v(x) \frac{1}{\lambda}+\sum_{y} p_{y} S(y)=\frac{v(x)+\sum_{y} \lambda_{y} S(y)}{\sum_{y} \lambda_{y}} \\
S(x) & =\frac{v(x)+\sum_{y} \lambda_{y} S(y)}{\lambda} .
\end{aligned}
$$

Here, $\lambda$ is the rate of departure from state $x . v(x)+$ $\sum_{y} \lambda_{y} S(y)$ is the QAST in state $x$ per time unit. This formula can be used to recursively evaluate the QAST in any stochastic tree model. It is called the rollback formula. However, one drawback of the formula is that it is risk neutral. For example, the intersystem soft handover between UMTS and DVB-H is not optimum when the duration is too short for the users that are staying in the handover destination network because a too-short duration can make the handover unnecessary and, sometimes, can cause a ping-pong effect. On the other hand, it is advisable to perform the intersystem soft handover between 
UMTS and DVB-H when the user pays a high price to get the optimum service quality or pays the lowest price, in spite of the poor service quality received. In this case, a risk factor $r(x)$ is introduced as the quality change when the user stays in network state $x$.

For a Markov process, $\lambda$ and $r(x)$ can always be assumed as exponential distributions [25]. Then, the QAST after considering risk factor $r(x)$ in state $x$ per time unit is given by

$$
R_{t}\left(x_{r}\right)=\int_{0}^{t}\left[v(x)+\sum_{y} \lambda_{y} S(y)\right] r(x) e^{-r(x) s} d s
$$

where $t$ is the time that the system spent in state $x$. From (15)

$$
R_{t}\left(x_{r}\right)=\left[v(x)+\sum_{y} \lambda_{y} S(y)\right]\left[1-e^{-r(x) t}\right] .
$$

Then, the QAST per time unit after considering risk factor $r(x)$ in state $x$ is given by

$$
R\left(x_{r}\right)=\frac{R_{t}\left(x_{r}\right)}{r(x)}=\frac{v(x)+\sum_{y} \lambda_{y} S(y)}{r(x)}\left[1-e^{-r(x) t}\right] .
$$

In this case, the total QAST after considering risk factor $r(x)$ is given by

$$
S\left(x_{r}\right)=\int_{0}^{\infty} R\left(x_{r}\right) \lambda e^{-\lambda t} d t=\frac{v(x)+\sum_{y} \lambda_{y} S(y)}{r(x)+\lambda} .
$$

When users perform the handover between UMTS and DVB-H, not only will risk happen but, sometimes, QAST can also be increased. For example, when users switch from UMTS to DVB-H, they benefit from the high-bit-rate advantages of DVB-H. On the other hand, when users switch from DVB-H to UMTS, they may benefit from the gained interaction characteristics of the service. Therefore, it is necessary to define a quality bonus variable $B(x)$ for the system.

After considering the quality bonus variable $B(x)$, the total QAST becomes

$$
S\left(x_{r}\right)=\frac{v(x)+\sum_{y} \lambda_{y}[B(y)+S(y)]}{r(x)+\lambda} .
$$

Since risk and bonus factors are always considered in the converged network systems, simply use $S(x)$ instead of $S\left(x_{r}\right)$ for the measurement QAST. Then

$$
S(x)=\frac{v(x)+\sum_{y} \lambda_{y}[B(y)+S(y)]}{r(x)+\lambda} .
$$

As $\lambda=\sum_{y} \lambda_{y}$, to make calculations easier and practical, (20) is written as

$$
S(x)=\frac{v(x)+\sum_{y} \lambda_{y}[B(y)+S(y)]}{r(x)+\sum_{y} \lambda_{y}} .
$$

The QAST is the measurement of the QoS of the system. Another measurement, i.e., COST, needs to be defined to evaluate the converged network operation cost. Similarly to the definition of the QAST, the COST is defined as being calculated by weighting each time interval $T_{x}$ spent in a particular network state $x$ by a network operation cost factor $c(x)$ proportional to the network operation cost that is estimated for that network state. Therefore, the COST is defined as

$$
\operatorname{COST}=\sum_{x} c(x) T_{x}
$$

where a network operation cost factor $c(x)=1$ refers to the maximum cost, whereas $c(x)=0$ refers to no cost; $c(x)$ takes values between 0 and 1 . Similarly to (21), the total COST for the converged network after considering risk and bonus factors can be calculated as

$$
C(x)=\frac{c(x)+\sum_{y} \lambda_{y}[B(y)+C(y)]}{r(x)+\sum_{y} \lambda_{y}}
$$

where $C(x)$ is the total COST, and $B(y)$ and $r(x)$ are the bonus and risk factors, respectively.

\section{Stochastic Tree Model for the Intersystem Soft Handover}

To use stochastic trees to model the intersystem soft handover for the converged network, the following assumptions are made.

1) The network state in the converged network is a Markovian property. This means that the next network state is only dependent on the present network state and has no dependence on the previous network states.

2) The service quality factor $v(x)$ and the network operation cost factor $c(x)$ do not depend on the number of users in the two networks; they are the statistical values that can be provided by the network operators.

3) The QAST is larger in a UMTS state than in a DVB-H state when a single user receives the same bit-rate service, but the service has an interactive nature.

4) The QAST is smaller in a UMTS state than in a DVB-H state when the number of users receiving the same service exceeds the preset threshold because of the congestion risk due to the unicast nature of UMTS.

5) The network operation cost COST is higher for DVB-H than for UMTS when the same service is delivered to a single user because the bandwidth is dedicated to one user in this case.

6) The network operation cost COST is higher for UMTS than for DVB-H when the same service is delivered to a certain number of users in the case that the user number exceeds the preset threshold because of the unicast nature of UMTS.

7) The soft handover state is a special state in the converged network. By default, it means the intersystem soft handover state between UMTS and DVB-H. The time the UE stays in the soft handover state is not negligible. The states after the soft handover state could be either 


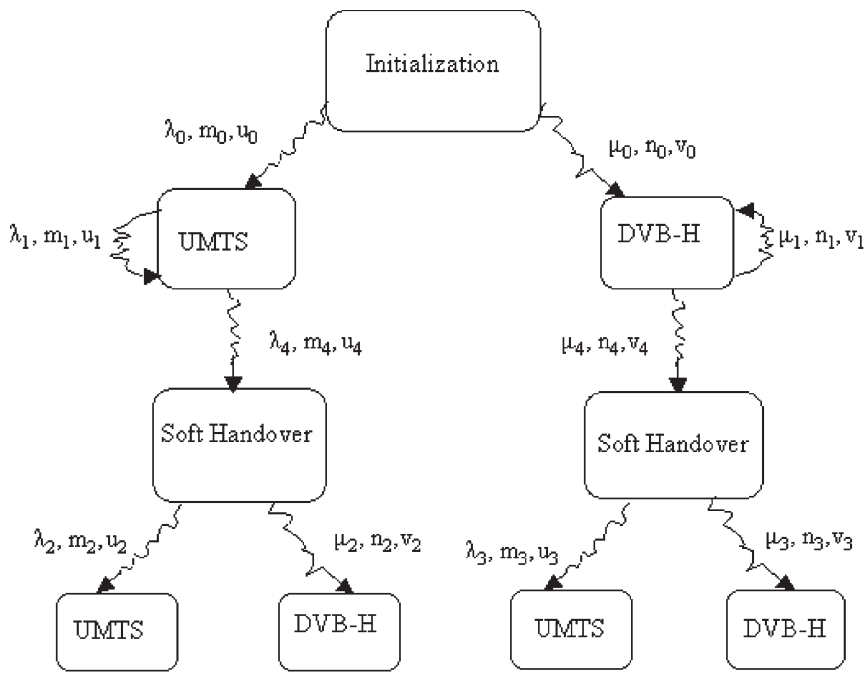

Fig. 12. Intersystem soft handover stochastic tree.

staying in the original state or entering into the handover destination state. The network operation cost is higher in the soft handover state than in the other states. The service quality is lower in the soft handover state than in the other states.

8) User-initiated intersystem soft handover is not permitted; therefore, the focus is on the optimum tradeoff between the network operation cost and the QoS from the network side.

Under these assumptions, a stochastic tree model is set up for the intersystem soft handover and is shown in Fig. 12. In Fig. 12, there are four different states. The transition probabilities are denoted by $\lambda_{i}$ and $\mu_{i}\left[i=0,1,2,3,4\right.$; here, $\lambda_{i}$ and $\mu_{i}$ have no relationships with those defined in (7) and (9)]. For each network state, the COST rates are denoted by $m_{i}$ and $n_{i}(i=0,1,2,3,4)$, and the QAST rates are denoted by $u_{i}$ and $v_{i}(i=0,1,2,3,4)$. Using (21) and (23), the expected total QAST and the expected total COST can be expressed as

$$
\begin{aligned}
E[Q(X)]= & \frac{v_{x}+\sum_{y} \lambda_{y}(B(y)+E[Q(y)])}{\beta_{x}+\sum_{y} \lambda_{y}} \\
E[C(X)]= & \frac{c_{x}+\sum_{y} \lambda_{y}(B(y)+E[C(y)])}{\alpha_{x}+\sum_{y} \lambda_{y}}
\end{aligned}
$$

where $X$ is one of the states in the stochastic tree, $c_{x}$ is the cost weight for state $X, v_{x}$ is the quality weight for state $X, \alpha_{x}$ is the state-specific cost discount rate at state $X$, and $\beta_{x}$ is the quality decrease attitude in state $X . B(y)$ is either COST rate $\left(m_{i}, n_{i}\right)$ or QAST rate $\left(u_{i}, v_{i}\right)$, depending on the network state. $E[Q(y)]$ and $E[C(y)]$ are the mean value of the COST and the QAST in the state after state $X$, respectively.

\section{Simulation and Results}

In this section, a numerical simulation and its analysis to find the optimum tradeoff between the converged network operation
TABLE I

\begin{tabular}{|c|c|c|}
\hline Description & $\begin{array}{c}\text { Variable } \\
\text { Name }\end{array}$ & Value \\
\hline \multirow{3}{*}{$\begin{array}{l}\text { From Initialization to } \\
\text { UMTS network probability } \\
\text { distribution }\end{array}$} & $\lambda_{0}$ & 0.5 \\
\hline & $\mathrm{m}_{40}$ & 0.2 \\
\hline & $u_{0}$ & 0.6 \\
\hline \multirow{3}{*}{$\begin{array}{l}\text { From Initialization to DVB- } \\
\mathrm{H} \text { network probability } \\
\text { distribution }\end{array}$} & $\mu_{0}$ & 0.5 \\
\hline & $\mathrm{n}_{0}$ & 0.8 \\
\hline & $\mathrm{vo}_{0}$ & 0.5 \\
\hline \multirow{3}{*}{$\begin{array}{l}\text { Soft handover probability } \\
\text { distribution from UMTS to } \\
\text { soft handover state }\end{array}$} & $\lambda_{4}$ & 0.5 \\
\hline & $\mathrm{m}_{4}$ & 1 \\
\hline & $\mathrm{u}_{4}$ & 0.4 \\
\hline \multirow{3}{*}{$\begin{array}{l}\text { Soft handover probability } \\
\text { distribution from DVB-H to } \\
\text { soft handover state }\end{array}$} & $\mu_{4}$ & 0.5 \\
\hline & $n_{4}$ & 1 \\
\hline & $v_{4}$ & 0.4 \\
\hline \multirow{3}{*}{$\begin{array}{l}\text { Probability distribution } \\
\text { from UMTS originated soft } \\
\text { handower state to UMTS }\end{array}$} & $\lambda_{2}$ & 0.5 \\
\hline & $m_{2}$ & 0.2 \\
\hline & $u_{2}$ & 0.6 \\
\hline \multirow{3}{*}{$\begin{array}{l}\text { Probability distribution } \\
\text { from UMTS originated soft } \\
\text { handover state to DVB-H }\end{array}$} & $\mu_{2}$ & 0.5 \\
\hline & $\mathrm{n}_{2}$ & 0.8 \\
\hline & $v_{2}$ & 0.5 \\
\hline \multirow{3}{*}{$\begin{array}{l}\text { Probability distribution } \\
\text { from DVB-H originated soft } \\
\text { handover state to UMTS }\end{array}$} & $\lambda_{3}$ & 0.5 \\
\hline & $m_{3}$ & 0.2 \\
\hline & $u_{3}$ & 0.6 \\
\hline \multirow{3}{*}{$\begin{array}{l}\text { Probability distribution } \\
\text { from DVB-H originated soft } \\
\text { handover state to DVB-H }\end{array}$} & $\mu_{3}$ & 0.5 \\
\hline & $n_{3}$ & 0.8 \\
\hline & $\mathrm{v}_{3}$ & 0.5 \\
\hline \multirow{3}{*}{$\begin{array}{l}\text { Probability distribution } \\
\text { staying in UMTS network } \\
\text { state }\end{array}$} & $\lambda_{1}$ & 0.5 \\
\hline & $\mathrm{m}_{1}$ & 0.2 \\
\hline & $\mathrm{ul}_{1}$ & 0.6 \\
\hline \multirow{3}{*}{$\begin{array}{l}\text { Probability distribution } \\
\text { staying in DVB-H network } \\
\text { state }\end{array}$} & $\mu_{1}$ & 0.5 \\
\hline & $\mathrm{n}_{1}$ & 0.8 \\
\hline & $\mathrm{vl}_{1}$ & 0.5 \\
\hline
\end{tabular}

Probabilities Used IN the Tradeoff ANALysis

cost COST and the QAST are presented. The simulation is done based on the stochastic tree structure shown in Fig. 12. From Fig. 12, for simplicity, it is reasonable to assume that the following equations are true:

$$
\begin{aligned}
m_{0} & =m_{1}=m_{2}=m_{3} \\
n_{0} & =n_{1}=n_{2}=n_{3} \\
m_{4} & =n_{4}=m_{0}+n_{0} \\
u_{0} & =u_{1}=u_{2}=u_{3} \\
v_{0} & =v_{1}=v_{2}=v_{3} \\
u_{4} & =v_{4} \leq \min \left\{u_{0}, v_{0}\right\} .
\end{aligned}
$$

Using (24) and (25), the expected total QAST and the expected total COST for UMTS and DVB-H can be calculated. Therefore, the tradeoff between the QAST and the COST can be obtained. Thus, the network side handover criteria can be set up based on the tradeoff between the converged network operation cost and the QAST.

The network scenario in the simulation is a hypertext transfer protocol (http) file delivery to a single user in the converged network. The http service can be individually received through the DVB-H and UMTS networks. Some interaction features are available if the service is delivered through UMTS. The variables and their values that are used in the numerical simulation are given. These parameters are shown in Table I. 


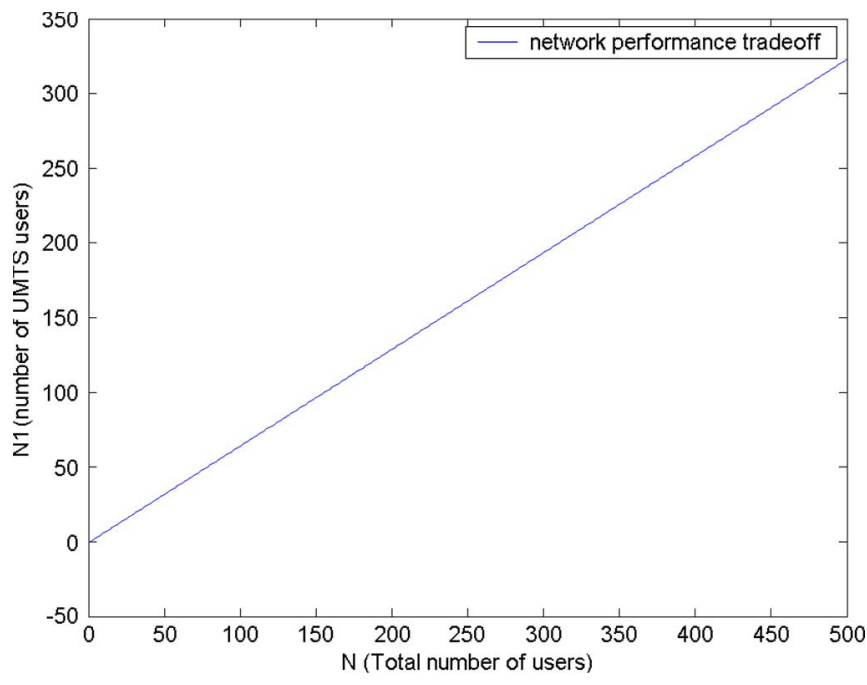

Fig. 13. Number of UMTS users versus number of total users.

For simplicity, it is also assumed that the risk factors $\alpha_{x}$ and $\beta_{x}$ are equal to zero. The mean values for the COST and the QAST in the last UMTS and DVB-H states of the stochastic tree are $E[Q(\mathrm{UMTS})]=u_{2}=u_{3}, E[Q(\mathrm{DVB}-\mathrm{H})]=v_{2}=v_{3}$, $E[C(\mathrm{UMTS})]=m_{2}=m_{3}$, and $E[C(\mathrm{DVB}-\mathrm{H})]=n_{2}=n_{3}$. For simplicity, the parameter values that are used in this simulation are very simple and are just for illustration; therefore, they may not reflect the real field test data. However, they are reasonable and can provide a general idea of how the algorithm works. Practical parameter values can be used in the model after real field tests in the network planning and optimization stage. From (24) and (25), the expected total COST and the expected total QAST for UMTS and DVB-H in the case of one user can be calculated along the two separate trunks of the stochastic tree as follows:

$$
\begin{aligned}
& E[C(X)]_{\mathrm{umts}}=5.4 ; E[C(X)]_{\mathrm{dvbh}}=3.6 \\
& E[Q(X)]_{\mathrm{umts}}=3.6 ; E[Q(X)]_{\mathrm{dvbh}}=3.3 .
\end{aligned}
$$

For $N$ users of the same service, suppose that $N_{1}$ users are using UMTS, and $N_{2}$ users are using DVB-H. The total COST and QAST for the converged network are $C_{\text {total }}=$ $5.4 N_{1}+3.6$ and $Q_{\text {total }}=3.6 N_{1}+3.3 N_{2}=0.3 N_{1}+3.3 N$, respectively. To find out the network performance tradeoff point, suppose that $C_{\text {total }}=Q_{\text {total }}$. Then, optimum number $N_{1}$ can be expressed as

$$
N_{1}=(11 N-12) / 17 .
$$

Tradeoff equation (26) is drawn in Fig. 13. Suppose that 500 users are served by the converged network with the same network parameters in the above simulation in the area. The optimum number of users that are served by a UMTS file delivery will be 323 .

Following the same simulation steps, more simulations can be performed; however, they are omitted because the simulation here is only used to illustrate the handover algorithms.

Using the presented stochastic tree model, the intersystem soft handover decision in the converged network can be made from technical and economic points of view. The model given above is not supposed to be complete; however, it gives a general idea of how to find the optimum tradeoff to perform intersystem handover in converged networks, where the technical issue is only one part of the problem, and the cost elements have to be considered. For different network planning and optimization strategies, different stochastic tree models can be set up.

\section{CONCLUSION}

A converged network on a common IP core is a trend for the next-generation communication systems. A DVB-H network and a UMTS network have different radio resource allocation processes and different handover mechanisms. In a converged DVB-H and UMTS network, cooperation between DVB-H and UMTS must happen to allocate radio resources and perform intersystem handover. This cooperation includes not only technical aspects that maximize the network performance and provide the best QoS to users but also economic aspects that maximize the operating profit for telecommunication and broadcast network operators.

This paper has proposed, for a converged DVB-H and UMTS network, a novel intersystem soft handover algorithm. To consider the intersystem handover criteria from technical and economic aspects, two new network measurement parameters, i.e., the QAST and the COST, were defined. Stochastic tree concepts were introduced, and a stochastic tree model for the converged network was presented. A numerical simulation was used to illustrate the intersystem soft handover process between DVB-H and UMTS. From the simulation results, the tradeoff between the network operation cost and the QAST can be found for the converged network.

\section{REFERENCES}

[1] ETSI EN 302304 V1.1.1, Digital Video Broadcasting (DVB); Transmission System for Handheld Terminals (DVB-H), Nov. 2004.

[2] DVB TM2967 (SB1299), Commercial Requirements: IP Datacast in DVB-H, Oct. 2003.

[3] J. Väre and M. Puputti, "Soft handover in terrestrial broadcast networks," in Proc. MDM, 2004, pp. 236-242.

[4] X. D. Yang, Y. H. Song, T. J. Owens, J. Cosmas, and T. Itagaki, "Seamless soft handover in DVB-H networks," in Proc. Softcom, 2004, pp. 27-30.

[5] M. Benson and H. J. Thomas, "Investigation of the UMTS to GSM handover procedure," in Proc. 55th IEEE VTC-Spring, May 6-9, 2002, vol. 4, pp. 1829-1833.

[6] D. Lugara, L. Ammi, M. Griguer, and J. Tartière, "UMTS to GSM handover based on compressed mode technique," in Proc. IEEE Int. Conf. Commun., Jun. 20-24, 2004, vol. 5, pp. 3051-3055.

[7] V. K. Varma, S. Ramesh, K. D. Wong, M. Barton, G. Hayward, and J. A. Friedhoffer, "Mobility management in integrated UMTS/WLAN networks," in Proc. IEEE Int. Conf. Commun., May 11-15, 2003, vol. 2, pp. 1048-1053.

[8] M. Jaseemuddin, "An architecture for integrating UMTS and 802.11 WLAN networks," in Proc. 8th IEEE Int. Symp. Comput. Commun., Jun. 30-Jul. 3, 2003, vol. 2, pp. 716-723.

[9] C. Skianis, G. Kormentzas, and K. Kontovasilis, "Interactions between intelligent multimodal terminals and a network management system in a B3G context," Wirel. Commun. Mob. Comput.-Special Issue on WLAN/3G Integration for Next-Generation Heterogeneous Networks, vol. 5, no. 6, pp. 679-695, 2005.

[10] D. Kouis, D. Loukatos, K. Kontovasilis, G. Kormenztas, and C. Skianis, "On the effectiveness of DVB-T for the support of IP-based services in heterogeneous wireless networks," Comput. Netw.-Special Issue on Internet Over Digital Broadcast Video Networks, vol. 48, no. 1, pp. 5773, 2005 . 
[11] A. K. Salkintzis, C. Fors, and R. Pazhyannur, "WLAN-GPRS integration for next-generation mobile data networks," Wireless Commun., vol. 9, no. 5, pp. 112-124, Oct. 2002.

[12] A. Centonza, G. A. Taylor, T. Itagaki, T. J. Owens, J. Cosmas, and Y. H. Song, "Stochastic user behaviour modelling and network simulation for resource management in cooperation with mobile telecommunications and broadcast networks," Int. J. Mobile Commun., vol. 4, no. 1, pp. 97$117,2006$.

[13] X. D. Yang, Y. H. Song, T. J. Owens, J. Cosmas, and T. Itagaki, "An investigation of and a proposal for handover decision-making in DVB-H," in Proc. 14th IST Mobile Wireless Commun. Summit, Dresden, Germany, Jun. 19-23, 2005.

[14] X. Lagrange, "Multitier cell design," IEEE Commun. Mag., vol. 35, no. 8, pp. 60-64, Aug. 1997.

[15] T.-S. P. Yum and W.-S. Wong, "Hot-spot traffic relief in cellular systems," IEEE J. Sel. Areas Commun., vol. 11, no. 6, pp. 934-940, Aug. 1993.

[16] 3GPP TS25.212, Multiplexing and Channel Coding (FDD), pp. 55-58, 2004-06. V6.2.0.

[17] ETSI, EN 300 744, Digital Video Broadcasting (DVB): Framing Structure, Channel Coding and Modulation for Digital Terrestrial Television, pp. 9-39, Nov. 2004. V1.5.1.

[18] J. Laiho, A. Wacker, and T. Novosad, Radio Network Planning and Optimization for UMTS. Hoboken, NJ: Wiley, 2002.

[19] T. Janevski, Traffic Analysis and Design of Wireless IP Networks. Norwood, MA: Artech House, 2003.

[20] H. Wu and C. Qiao, "Modeling iCAR via multi-dimensional Markov chains," Mobile Netw. Appl., vol. 8, no. 3, pp. 295-306, Jun. 2003.

[21] K. Mitchell and K. Sohraby, "An analysis of the effects of mobility on bandwidth allocation strategies in multi-class cellular wireless networks," in Proc. INFOCOM, Apr. 22-26, 2001, vol. 2, pp. 1005-1011.

[22] G. Hazen, "Stochastic trees: A new technique for temporal medical decision modeling," Med. Decis. Mak., vol. 12, no. 3, pp. 163-178, Jul.-Sep. 1992.

[23] G. Hazen, "Preference factoring for stochastic trees," Manag. Sci., vol. 46, no. 3, pp. 389-403, Mar. 2000.

[24] G. Hazen, "Factored stochastic trees: A tool for solving complex temporal medical decision models," Med. Decis. Mak., vol. 13, no. 3, pp. 227-236, 1993.

[25] G. B. Hazen and J. M. Pellissier, "Recursive utility for stochastic trees," Oper. Res., vol. 44, no. 5, pp. 788-809, Sep./Oct. 1996.

[26] [Online]. Available: www.ist-instinct.org/

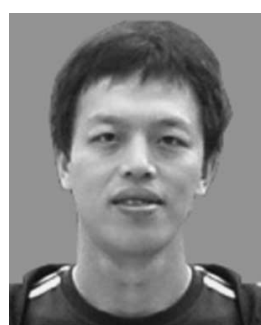

Xiaodong Yang received the B.E. degree from Tianjin University, Tianjin, China, in 1999, the M.E. degree from Tsinghua University, Beijing, China, in 2002, and the Ph.D. degree from Brunel University, Uxbridge, U.K., in 2005.

From February 2006 to June 2007, he was a Postdoctoral Researcher with the Institute for Informatics, University of Göttingen, Göttingen, Germany. $\mathrm{He}$ was also with Hutchison $3 \mathrm{G}$ Austria $\mathrm{GmbH}$, Vienna, Austria, working for the DVB-H project, and is serving as a Guest Lecturer with the Computer Networks Group, University of Göttingen. His research interests include broadcast networks (DVB-T/H, etc.), telecommunications networks (GSM/UMTS, etc.), and IP networks.

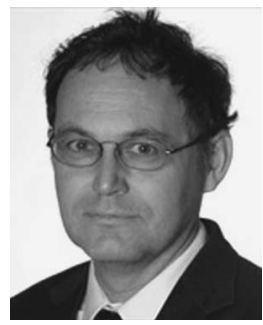

Thomas J. Owens received the Ph.D. degree in electrical and electronic engineering from the University of Strathclyde, Glasgow, U.K., in 1986.

In 1987, he joined Brunel University, Uxbridge, U.K., where he is a Senior Lecturer with the School of Engineering and Design. He is the author of more than 30 refereed journal papers and book chapters in the areas of engineering and applied mathematics.

Mr. Owens is on the Editorial Advisory Board of the International Journal of Engineering Education and on the Editorial Board of the International Journal of Services and Standards. He was the Project Coordinator of the Information Society Technologies (IST) Fifth Framework Programme Project CONFLUENT and the IST Sixth Framework Programme Integrated Project INSTINCT. He is a Chartered Engineer and a Chartered Mathematician. 